\title{
Sobrepeso y obesidad en un grupo de escolares españoles
}

\author{
Overweight and obesity in a group \\ of spanish school age children
}

\begin{abstract}
Objetive: To evaluate the prevalence of overweight and obesity in a sample of Spanish schoolchildren by gender. Material and methods: The study involved 505 schoolchildren (259 boys and 246 girls), aged between 8 and 13 years (4th, 5th and 6th grade) from various Spanish provinces: A Coruna, Barcelona, Madrid, Seville and Valencia. Health, socioeconomic, anthropometric data (weight and height) and physical activity were collected and body mass index was calculated. Results: The prevalence of overweight was 15,9\%, being higher in girls (21,9\%) than in boys $(10,1 \%)$, and obesity was $17,3 \%$, being higher in boys $(24,5 \%)$ than in girls $(9,7 \%)$. A higher percentage of fat $(36.9 \%)$ measured by arm fat area (AFA\%) was found in girls. linstead boys had more central adiposity by applying the waist-hip ratio $(0,86 \pm 0,05)$. Conclusions: The schoolchildren studied are in alarming situation regarding the condition of overweight and obesity. Even, the situation has worsened compared to data from previous studies, which highlights the importance of controlling these parameters from an early age.

Key words: Overweight, obesity, prevalence, school, Spain.
\end{abstract}

Alexia De Piero (1)

Elena Rodríguez-Rodríguez (2)

Liliana Guadalupe González-Rodríguez (3)

Ana María López-Sobaler (3)

(1) Instituto Superior de Investigaciones Biológicas (INSIBIO), FacultaddeBioquímica, Química y Farmacia, Universidad Nacional deTucumán. Argentina. (2) Sección Departamental de Química Analítica, Facultad de Farmacia, Universidad Complutense de Madrid, Ciudad Universitaria, Madrid, España. (3) Departamento de Nutrición, Facultad de Farmacia, Universidad Complutense de Madrid, Ciudad Universitaria, Madrid, España.

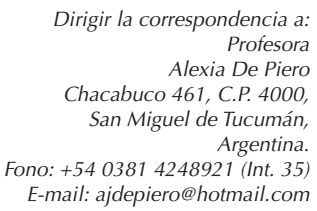

Este trabajo fue recibido el 24 de Febrero de 2014 y aceptado para ser publicado el 15 de Julio de 2014.

\section{INTRODUCCIÓN}

La obesidad es una enfermedad nutricional caracterizada por el aumento de la grasa corporal constituyendo hoy día un problema de salud pública mundial (1) con importantes repercusiones sobre la salud, ya que se ha relacionado con el padecimiento de enfermedades como la diabetes, hipertensión, dislipemias, enfermedad cardiovascular y algunos tipos de cáncer (2).

Su prevalencia es especialmente preocupante en la infancia y niñez, ya que se ha producido un aumento en las últimas décadas. En relación a los datos disponibles a nivel mundial, la tendencia secular de obesidad en EEUU ha ido aumentando a un ritmo alarmante. De acuerdo con los datos del National Health and Nutrition Examination Surveys (NHANES I-IV) en 1999 el porcentaje de niños de 6 a 11 años con un índice de masa corporal (IMC) superior al percentil 95 era $13 \%$ y entre los jóvenes de 12 a 19 14\%. Esta situación demostró un aumento con relación a las cifras del período 2009-2010 donde el predominio de obesidad fue 16,9\% en niños y adolescentes (3). Las estimaciones actuales indican que el $17 \%$ de los niños y adolescentes presentan obesidad, lo que representa un aumento de tres veces en comparación con hace tres décadas (4). Sin embargo, entre 1999-2000 y 2009-2010, no hubo una tendencia significativa en la incidencia de obesidad en las niñas, a pesar de haberse observado un aumento significativo en los niños (3). Recientes estudios revelan que la tendencia de sobrepeso en niños de edad escolar alcanza 35\% en algunas partes de Europa, al mismo tiempo que diversos países incrementan anualmente la incidencia de nuevos casos (5).

Los datos en España indican un aumento en la prevalencia de obesidad del 4,9\% en niños de ambos sexos, en 1984 (estudio Paidos) y hasta un aumento en la prevalencia de sobrepeso de $12,5 \%$ y obesidad de 13,9\% en el año 2000 (estudio enKid), y de $14 \%$ y $16,8 \%$, respectivamente, según el estudio ALADINO. El estudio ALADINO es el estudio más reciente realizado en la población infantil-española, aporta datos de prevalencia de sobrepeso y obesidad en escolares de 6 a 9 años de edad (6).

La obesidad infantil y las comorbilidades asociadas en la actualidad predicen en Europa un gasto sanitario de unos 10 billones de euros anuales (7). En España el costo de la obesidad supone $7 \%$ del gasto sanitario, cifra elevada con respecto a países vecinos, como Francia o Portugal, donde representa entre $2 \%$ y $3,5 \%$ del gasto sanitario (8).

El objetivo de este estudio fue evaluar la prevalencia de sobrepeso y obesidad en un grupo de escolares españoles con edades comprendidas entre 8 y 13 años. 


\section{SUJETOS Y MÉTODOS \\ Sujetos}

Se estudió una muestra de conveniencia de niños y niñas españoles, de 8 a 13 años de edad, que realizaban sus estudios de educación primaria (EP) en colegios públicos de 5 localidades españolas (A Coruña, Barcelona, Madrid, Sevilla y Valencia). El estudio se realizó en 10 centros escolares públicos. La selección de los colegios se llevó a cabo por nuestro equipo investigador, teniendo en cuenta los siguientes requisitos, pertenecer a la provincia seleccionada, ser colegios públicos de educación primaria y encontrarse dentro de zonas residenciales, para que los escolares fueran de un nivel socioeconómico similar. En cada localidad se contactó dos colegios, y tras la presentación del estudio al director del centro y al consejo escolar, se organizó una reunión con los padres y tutores de los escolares pertenecientes a los cursos de $4^{\circ} \mathrm{a}$ $6^{\circ}$ de EP, explicándoles en detalle el estudio y solicitando su consentimiento informado para la participación de los niños en el estudio. Se eligieron niños de $4^{\circ}, 5^{\circ}$ y $6^{\circ}$ de EP porque a esta edad tienen capacidad suficiente para contestar sin ayuda algunos de los cuestionarios y preguntas planteadas en la investigación general. La muestra inicial estuvo integrada por los escolares que cumplieron con los siguientes criterios de inclusión, participación voluntaria y autorización firmada de los padres o tutores legales de los escolares, edad comprendida entre 8 y 13 años de edad, libres de enfermedades (endocrinas, metabólicas) y/o estar tomando habitualmente fármacos que pudieran modificar la ingesta o utilización de los nutrientes. El criterio de exclusión fue la inasistencia al centro los días concertados para hacer las pruebas o entrevistas.

La muestra no fue representativa de los escolares españoles, sin embargo nos permite analizar los hábitos alimentarios, la situación nutricional y algunos datos sanitarios y antropométricos de los escolares españoles.

El protocolo del presente estudio fue aprobado por el Comité Ético de Investigación de la Facultad de Farmacia, de la Universidad Complutense de Madrid.

\section{Métodos}

A los escolares que aceptaron participar en el estudio se les aplicaron diferentes cuestionarios, que fueron completados por los padres o tutores. Se concertó con el centro escolar y con los padres de los escolares los días en los que se llevarían a cabo los distintos estudios y se explicaron los requisitos necesarios para la realización de los mismos.

La antropometría incluyó el peso y la talla determinados por báscula digital electrónica (modelo Seca Alpha; rango: 0,1150 kg; precisión 100 g) y un estadiómetro digital Harpenden (Pfifter, Carlstadt, NJ, USA; range 70-205 cm, precisión $1 \mathrm{~mm}$ ), respectivamente. Todos los datos se registraron siguiendo las normas de la OMS (9). El Índice de Masa Corporal (IMC) se calculó a partir de las medidas de peso y talla $(\mathrm{IMC}=$ peso [kg]/talla [m2]) y se utilizó para clasificar a la población según su estado ponderal estableciéndose los siguientes criterios de Hernández para la población de referencia (10) (delgadez <P25; normopeso >P25 y <P85; sobrepeso >P85 y <P97; obesidad $>$ P97).

A partir del IMC se calculó: Puntuación Z o "puntaje Z" otra manera de interpretar los datos obtenidos es haciendo uso de la puntuación típica (P.T. o puntaje Z), que permite conocer el múltiplo o fracción de desviaciones estándar que un individuo se separa de la media; este puntaje $Z$ es obtenido de los valores de las medias o medianas y de las desviaciones estándar (del patrón de referencia ó estándar) (Z-IMC= [A-B]/DS).
Se tomó la circunferencia corporal con una cinta métrica inextensible de acero marca Holtain (rango: 0-150 cm; precisión de $1 \mathrm{~mm}$ ), las mediciones de las circunferencias se realizaron en centímetros:

Circunferencia de cintura: Se midió entre la línea directa entre el margen costal inferior y la cresta ilíaca (espina ilíaca anterosuperior) en bipedestación (11). Se clasificaron a los niños de acuerdo con la presencia o no de obesidad central con respecto a la circunferencia de cintura ( $>$ P90).

Circunferencia de la cadera: Ésta medida se realizó con el escolar de pie, tomada en el punto de máxima circunferencia sobre los glúteos, colocando la cintra métrica de manera horizontal al suelo (9).

Circunferencia del brazo: se midió, en primer lugar, la longitud del brazo derecho, que se tomó con el niño de pie y con el codo flexionado. Se midió la distancia entre la punta del hombro (acromion) y la cabeza del radio (olécranon). Con el brazo extendido, se midió la circunferencia en la parte media del brazo, con una cinta métrica en posición horizontal. Se midió para estimar la proporción de grasa y masa magra del cuerpo (12).

Se midió el pliegue cutáneo tricipital (PT) como indicador de la masa grasa corporal. Para su medición se utilizó un caliper (marca HOLTAIN LTD. CRYMYCH UK, de presión constante y de $10 \mathrm{~g} / \mathrm{mm} 2$ de superficie de contacto [rango 0-39 mm; precisión 0,2 $\mathrm{mm}$ ]. Se realizó en el lado derecho del cuerpo, siguiendo las normas de la OMS (9), expresando la medida en milímetros $(\mathrm{mm})$

A partir de estos parámetros se calcularon los siguientes indicadores de composición corporal:

Índice cintura/cadera (IC/Ca): Proporciona información sobre la distribución de la grasa corporal. Es un indicador de adiposidad central que diferencia entre la obesidad androide y la obesidad ginecoide, siendo también válida su utilización en niños (13). Se calculó mediante la siguiente fórmula (IC/ $\mathrm{Ca}=$ circunferencia de cintura $[\mathrm{cm}] /$ circunferencia de cadera $[\mathrm{cm}])$.

Índice cintura/altura: Es de fácil obtención y tiene como ventaja valores únicos de referencia independientemente del sexo y la edad. Es un buen indicador de la adiposidad, y de riesgo cardiovascular en adultos y niños. Se consideran con riesgo los niños con un índice mayor a 0,5 (14-16). Se calculó según la siguiente fórmula (ICA= circunferencia de la cintura $[\mathrm{cm}] /$ altura[cm]).

Se analizó la adecuación de las reservas grasas y proteicas, para ello se calcularon las áreas grasas y musculares braquiales a partir de las siguientes fórmulas (Área grasa del brazo (AGB)= $\mathrm{C} 2 /[4 \pi]) ;($ Área muscular del brazo $(\mathrm{AMB})=[\mathrm{C}-(\mathrm{PT} \pi)] 2 /(4 \pi)$ ); (Área grasa del brazo $(A G B)=A T B-A M B)$. Como referencia se consideraron los valores de $A M B$ y $A G B$ (considerando el P50 como punto de referencia) de Marrodán y col. (17).

Actividad física: Para conocer el grado de actividad física que realizaban los escolares y poder determinar su coeficiente de actividad se solicitó a los padres completar un cuestionario (18), donde se registraban diversas actividades, debiendo indicar el tiempo diario (en una media de 24 horas) dedicado a cada actividad (dormir, comer, jugar, entre otras). Con los datos obtenidos se estableció el tiempo (horas) dedicado al reposo, y a la realización de actividades muy ligeras, ligeras y moderadas. Las horas empleadas para cada actividad se multiplicaron por los factores correspondientes a reposo por 1, actividad muy ligera por 1,5 (actividades que pueden realizarse sentado o estando de pie), actividad ligera por 2,5 (pasear) y moderadamente activos por 5 (bailar o jugar al tenis), cuya 
suma y división posterior por 24 permitió obtener un coeficiente de actividad individual, reflejo del grado de actividad desarrollada para cada escolar (19). El resultado obtenido es el coeficiente de actividad individualizado (CAFI), a partir del cual se clasifican las categorías de actividad en sedentario $(1,0-$ $<1,4)$, poco activo $(1,4-<1,6)$, activo $(1,6-<1,9)$ y muy activo $(1,9-<2,5)$, con el cual se obtiene el coeficiente de actividad física, se estima el gasto energético (20).

\section{ANÁLISIS ESTADÍSTICO}

Los datos se procesaron utilizando el software Rsigma Babel (Horus Hardward, Madrid). Se consideraron datos personales, antropométricos y de actividad física en función del sexo. Se determinaron valores medios, desviación estándar (DS), y porcentajes (\%) para cada uno de los parámetros estudiados. Para la significación de las diferencias entre medias, se utilizó el test de la "t" de Student. En el caso de pruebas no paramétricas con distribución no homogénea mediante el test de Mann-Whitney. Para establecer la asociación entre dos variables, cuando los datos presentaron una distribución normal se utilizó la correlación de Pearson. Cuando los datos no siguieron una distribución normal o la variable independiente fue cualitativa se utilizó la correlación de Spearman. Los valores OR se utilizaron para comparar la frecuencia con que ocurre un efecto entre los que estan expuestos al factor de riesgo y los que no lo están, indicando la probabilidad de que ocurrael suceso en el primer grupo frente al segundo. Se consideraron significativas las diferencias con $p<0,05$.

\section{RESULTADOS}

En la tabla 1 se presentan los datos personales y antropométricos de los niños objeto de estudio. La muestra fue de 505 escolares, de los cuales 259 fueron niños $(51,2 \%)$ y 246 niñas (48,7\%), con edades entre los 8 y 13 años con

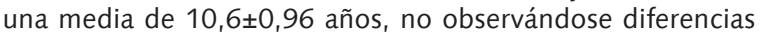
estadísticamente significativas de edad, peso, talla o IMC en cuanto al sexo. La prevalencia de sobrepeso y obesidad fue $15,9 \%$ y $17,3 \%$, respectivamente, siendo mayor el sobrepeso en niñas (21,9\%) y la de obesidad en niños (24,5\%). En cuanto a los índices de distribución de la grasa corporal, tanto el IC/ Ca como el ICA fueron significativamente superiores en los varones, además el riesgo cardiovascular fue menor, por ser la media del ICA (relación cintura/altura $<0,5$ ). El AGB y $A M B$ fue similar en niños y niñas, siendo significativamente superior el AGB (\%) en las niñas.

En la tabla 2 se presentan los datos de actividad física de los niños. Se observaron diferencias significativas en el tiempo dedicado a actividades sedentarias en niños y niñas $(p<0,05)$. Los niños con actividad de tipo sedentario presentaron mayor IMC puntaje $Z$ a diferencia de las niñas (tabla 3). Al realizar un análisis de regresión logística, se observó que los escolares más activos presentaban una menor probabilidad

TABLA 1

Datos antropométricos de los escolares según sexo.

\begin{tabular}{|c|c|c|c|c|}
\hline & $\begin{array}{c}\text { Total } \\
(n=505)\end{array}$ & $\begin{array}{c}\text { Niños } \\
(n=259)\end{array}$ & $\begin{array}{c}\text { Niñas } \\
(n=246)\end{array}$ & $\mathrm{p}$ \\
\hline Edad (años) & $10,6 \pm 0,96$ & $10,5 \pm 0,99$ & $10,6 \pm 0,92$ & - \\
\hline Peso (kg) & $40,0 \pm 9,5$ & $39,8 \pm 9,5$ & $40,2 \pm 9,6$ & - \\
\hline Talla $(\mathrm{cm})$ & $143,8 \pm 8,6$ & $143,1 \pm 8,3$ & $144,5 \pm 8,8$ & - \\
\hline $\mathrm{I} M C\left(\mathrm{~kg} / \mathrm{m}^{2}\right)$ & $19,1 \pm 3,1$ & $19,2 \pm 3,1$ & $19,0 \pm 3,0$ & - \\
\hline TMB (kcal/día) & $1310,0 \pm 171,1$ & $1346,7 \pm 194,1$ & $1272,0 \pm 133,5$ & ${ }^{* * *} \dagger$ \\
\hline GET (kcal/día) & $2106,8 \pm 448,8$ & $2188,8 \pm 446,0$ & $2021,4 \pm 436,4$ & $* * *$ \\
\hline \multicolumn{5}{|l|}{ Valoración ponderal (\%) } \\
\hline Delgadez & 28,0 & 26,8 & 29,2 & - \\
\hline Normal & 38,7 & 38,5 & 39,0 & - \\
\hline Sobrepeso & 15,9 & 10,1 & 21,9 & $* * *$ \\
\hline Obesidad & 17,3 & 24,5 & 9,7 & $* * *$ \\
\hline Puntaje Z del IMC & $0,66 \pm 1,3$ & $0,83 \pm 1,4$ & $0,47 \pm 1,2$ & ${ }^{* *}+$ \\
\hline Circ. de cintura $(\mathrm{cm})$ & $67,1 \pm 8,9$ & $67,6 \pm 9,2$ & $66,5 \pm 8,5$ & - \\
\hline Circ. de cadera $(\mathrm{cm})$ & $78,4 \pm 8,7$ & $77,7 \pm 8,6$ & $79,2 \pm 8,8$ & - \\
\hline $\mathrm{IC} / \mathrm{Ca} 0,85 \pm 0,05$ & $0,86 \pm 0,05$ & $0,83 \pm 0,05$ & $* * *$ & \\
\hline ICA $\quad 0,46 \pm 0,05$ & $0,47 \pm 0,05$ & $0,46 \pm 0,05$ & * & \\
\hline Circ. del brazo (cm) & $22,8 \pm 3,2$ & $22,8 \pm 3,3$ & $22,8 \pm 3,1$ & - \\
\hline Pliegue tricipital (mm) & $14,7 \pm 5,9$ & $14,1 \pm 6,4$ & $15,4 \pm 5,3$ & ${ }^{*} \dagger$ \\
\hline $\mathrm{AGB}\left(\mathrm{cm}^{2}\right)$ & $15,7 \pm 7,7$ & $15,1 \pm 8,3$ & $16,2 \pm 7,1$ & - \\
\hline AGB (\%) & $35,3 \pm 9,3$ & $33,7 \pm 10,1$ & $36,9 \pm 7,9$ & $*^{* *} \dagger$ \\
\hline $\mathrm{A} M \mathrm{~B}\left(\mathrm{~cm}^{2}\right)$ & $26,7 \pm 6,0$ & $27,3 \pm 6,1$ & $26,2 \pm 5,9$ & * \\
\hline
\end{tabular}


de tener elevado IMC puntaje Z [OR=1,3739 (1.0924-1,7281); $\mathrm{p}<0,001)$. En el análisis de regresión lineal de Pearson no se observaron relación estadísticamente significativa entre el CAFI y el IMC puntaje $Z(r=0,087416 ; p>0,05)$. Al realizar el análisis de regresión lineal de Pearson no se encontró relación entre el IMC puntaje $Z$ ni el hecho de ser sedentario $(r=0,046846 ; p>0,05)$, poco activo $(r=0,03237 ; p=>0,05) o$ activo $(r=0,046846 ; p=>0,05)$.

\section{DISCUSIÓN}

El presente estudio aporta datos recientes sobre el estado nutricional de una muestra de escolares españoles.

En la antropometría, no se hallaron diferencias estadísticamente significativas con respecto al sexo para los valores de peso, talla o IMC, situación esperable por tratarse de una población bastante homogénea, con un crecimiento y desarrollo adecuado. Otros estudios han encontrado diferencias, así el estudio de López-de Lara y col. (21), en una muestra comprendida entre 3 y 24 años de ambos sexos, encontraron mayor peso en niñas de 8,5 años y en niños de 5,8 años. También observaron diferencias en estos parámetros ÁlveroCruz y col. (22) en un grupo de niños de 12 a 18 años de la ciudad de Málaga, donde hubo diferencias significativas en el peso y la talla, con valores mayores en niños que en niñas. En el estudio de López-de Lara y col. (21), el IMC fue mayor en niños de 11 años de edad con respecto a las niñas.

Si bien la población estuvo integrada por una muestra de escolares sanos, se observó un marcado sedentarismo en los

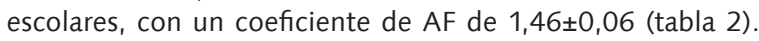
Los varones dedicaron más tiempo a actividades de ocio como jugar con el ordenador y videoconsola, aunque estas actividades sedentarias se compensaban con mayor tiempo destinado a la práctica de actividades extraescolares deportivas. Esto explica un coeficiente de actividad final ligeramente superior a las niñas, que utilizaron más horas del día en actividades más sedentarias, lo que explica que el GET de la muestra femenina

TABLA 2

Actividad física de los escolares. Diferencias según el sexo.

\begin{tabular}{|c|c|c|c|c|}
\hline & $\begin{array}{c}\text { Total } \\
(n=505)\end{array}$ & $\begin{array}{c}\text { Niños } \\
(n=259)\end{array}$ & $\begin{array}{c}\text { Niñas } \\
(n=246)\end{array}$ & $\mathrm{p}$ \\
\hline \multicolumn{5}{|c|}{ Tiempo dedicado a diferentes actividades (h/día): } \\
\hline Dormir & $9,3 \pm 0,72$ & $9,3 \pm 0,75$ & $9,3 \pm 0,67$ & - \\
\hline Ver televisión & $1,4 \pm 0,82$ & $1,4 \pm 0,79$ & $1,3 \pm 0,85$ & - \\
\hline Caminar & $0,32 \pm 0,28$ & $0,31 \pm 0,28$ & $0,32 \pm 0,28$ & - \\
\hline Ordenador y video consola & $0,59 \pm 0,52$ & $0,65 \pm 0,49$ & $0,53 \pm 0,54$ & * \\
\hline Práctica deportiva total & $0,62 \pm 0,37$ & $0,68 \pm 0,35$ & $0,55 \pm 0,37$ & ** \\
\hline Educación física en el colegio & $0,24 \pm 0,02$ & $0,24 \pm 0,02$ & $0,24 \pm 0,02$ & - \\
\hline Práctica deportiva extraescolar & $0,38 \pm 0,37$ & $0,43 \pm 0,35$ & $0,31 \pm 0,37$ & ** \\
\hline Actividades de reposo & $9,3 \pm 0,72$ & $9,3 \pm 0,75$ & $9,3 \pm 0,67$ & - \\
\hline Actividades muy ligeras & $18,2 \pm 1,6$ & $18,1 \pm 1,5$ & $18,4 \pm 1,6$ & - \\
\hline Actividades ligeras & $4,5 \pm 2,2$ & $4,6 \pm 2,3$ & $4,4 \pm 2,2$ & - \\
\hline Actividades moderadas & $3,1 \pm 1,8$ & $3,4 \pm 1,7$ & $2,7 \pm 1,8$ & ** \\
\hline Coeficiente de actividad física individual & $1,46 \pm 0,06$ & $1,47 \pm 0,07$ & $1,45 \pm 0,06$ & ** \\
\hline
\end{tabular}

TABLA 3

Actividad física categorizada de los escolares. Diferencias según el IMC puntaje Z y sexo.

\begin{tabular}{|c|c|c|c|}
\hline & \multicolumn{2}{|c|}{ IMC Puntaje Z } & \multirow[b]{2}{*}{$p$} \\
\hline & Niños & Niñas & \\
\hline Sedentario & $0,67 \pm 0,92$ & $0,15 \pm 0,94$ & $*$ \\
\hline Poco activo & $0,92 \pm 1,4$ & $0,59 \pm 1,1$ & $-\dagger$ \\
\hline Activo & $0,31 \pm 1,2$ & $-0,31 \pm 1,1$ & - \\
\hline
\end{tabular}


$(2022,4 \pm 351,8 \mathrm{kcal})$ fue menor (tabla 1$)$. Estos resultados son similares a los de Velasco y col. (23), en donde el gasto energético fue mayor en niños. Los escolares estudiados no presentaron diferencias significativas en la actividad física en función de su situación ponderal (tabla 3) ( $r=0,03237)$, a pesar de que se observó diferencias en la actividad física en función del sexo, la prevalencia de obesidad fue mayor en los niños. Esta situación fue similar a la observada en otros estudios que han indicado que los niños con sobrepeso son menos activos que los con peso normal (24).

La Organización Mundial de la Salud ha propuesto el IMC como parámetro para el diagnóstico de sobrepeso y obesidad, ya que muestra una buena correlación con la adiposidad total y una fuerte correlación epidemiológica con la morbimortalidad asociada a la obesidad en adultos (25). Además, es un predictor de riesgo cardiovascular en niños, a pesar de que en la actualidad se ha demostrado que existen mejores predictores, como son las medidas de circunferencia de cintura y el índice cintura/talla (26), razón por la que se determinaron en este trabajo.

Se observó en dicha muestra una elevada incidencia de sobrepeso $(15,9 \%)$ y de obesidad (17,3\%), medidas por el IMC según el criterio de Hernández y col. (10), siendo mayor el sobrepeso en niñas y la obesidad en niños $(p<0,001)$ (tabla 1).

Al comparar estos resultados con los de otras poblaciones infanto-juveniles, a pesar de existir consenso en cuanto a definirlos en función del IMC, existe una diversidad de criterios diagnósticos, con respecto a los puntos de referencia empleados para definir sobrepeso y obesidad.

En España, en los estudios realizados por Álvarez y col. (27); Durá y col. (28); Sánchez-Cruz y col. (29); García y col. (30) y Amigo y col. (31), encontraron una mayor prevalencia de sobrepeso y menor de obesidad que los encontrados en nuestro estudio. En el estudio realizado por Durá y col. (28), observaron que $32,6 \%$ de la población presentó sobrepeso, y en el estudio de García y col. (30), observaron que 9,5\% de la población presentó obesidad, siguiendo el criterio del IOTF
(International Obesity Task Force), que es menos restrictivo que el de Hernandez y col. (10) debido a que utilizan diferentes criterios (29) [Hernández y col. (10) definen sobrepeso cuando P>85 y obesidad cuando P>97; el IOTF (29) define sobrepeso cuando el IMC $>25 \mathrm{~kg} / \mathrm{m}^{2}$ y obesidad cuando el IMC >30 kg/m² en población infantil]. Del mismo modo, se observó que en los estudios realizados en Estados Unidos por Ogden y col. (55), y Rossen (32), una mayor prevalencia de sobrepeso y de obesidad que en nuestro estudio, siguiendo el criterio de NHANES (National Health and Nutrition Examination Survey) (30).

Las diferencias encontradas en los porcentajes de sobrepeso y obesidad pueden deberse a los rangos de edad de cada muestra, tipos de muestras, definición de los puntos de corte y a los criterios de referencia utilizados en cada estudio (cuadro 1). La incidencia de sobrepeso y obesidad encontrada en este estudio fue similiar al estudio enKid, y esta semejanza podría deberse al hecho de haber empleado el mismo criterio para definir sobrepeso y obesidad, ya que en ambos estudios se usaron las tablas de Hernández y col. (10) como referencia para establecer los puntos de corte de sobrepeso y obesidad.

Cabe destacar que, aunque para el IMC no encontramos diferencias con respecto al sexo, para el caso del puntaje $Z$ del IMC, si se encontraron diferencias entre sexos, siendo superior en niños que en niñas (tabla 1), lo que contrasta con el estudio realizado en una población de entre 8 y 18 años por Heo y col. (33), que observaron que el puntaje Z del IMC fue similar en niños y niñas. Con respecto a nuestro estudio, sería de esperar que las niñas presentaran mayor puntaje $Z$ del IMC debido al rebote adiposo acompañado de incremento adiposo, por lo contrario, hemos observado que en los niños es superior éste índice, lo que podría suponer que los niños presentan una dieta desequilibrada por presentar un patrón alimentario hipercalórico, hiperproteico, con alto contenido graso y bajo en hidratos de carbono $(34,35)$, además de existir asociación entre la situación ponderal de los padres con la situación ponderal de los niños, como se ha demostrado en

\section{CUADRO 1}

Prevalencia de sobrepeso y obesidad en escolares y adolescentes.

\begin{tabular}{|c|c|c|c|}
\hline Edad en años & País o ciudad & Porcentaje & Referencia \\
\hline 8-17 años & España & $\begin{array}{l}26 \% \text { sobrepeso } \\
12,6 \% \text { obesidad }\end{array}$ & Sánchez-Cruz y col., 2013 (51) \\
\hline 8-13 años & & $45 \%$ exceso de peso & \\
\hline 2-16 años & Almería & $\begin{array}{l}22,4 \% \text { sobrepeso } \\
9,5 \% \text { obesidad }\end{array}$ & García y col., 2013 (52) \\
\hline 6-14 años & $\begin{array}{l}\text { España } \\
11,6 \% \text { obesidad }\end{array}$ & $24,6 \%$ sobrepeso & Coronado y col., 2012 (53) \\
\hline 6-11 años & Alicante & $\begin{array}{c}9,71-22,3 \% \text { sobrepeso } \\
8,9-18,8 \% \text { obesidad }\end{array}$ & Ruíz y col., 2008 (54) \\
\hline 2-19 años & EEUU & $\begin{array}{l}17,1 \% \text { sobrepeso } \\
32,2 \% \text { obesidad }\end{array}$ & Ogden y col., 2006 (55) \\
\hline 2-14 años & Madrid & $\begin{array}{l}17,4 \% \text { sobrepeso } \\
13,2 \% \text { obesidad }\end{array}$ & Albañil y col., 2005 (56) \\
\hline 5-18 años & Nigeria & $\begin{array}{l}11,4 \% \text { sobrepeso } \\
2,8 \% \text { obesidad }\end{array}$ & Ene-Obong y col., 2012 (57) \\
\hline
\end{tabular}


otros estudios $(31,36)$.

Para valorar la composición corporal en este estudio, se utilizó la medición del pliegue cutáneo de tríceps, por su utilidad de reflejar de manera adecuada el grado de adiposidad total del individuo (37). Durante la edad escolar se producen cambios mayores que los observados en etapas anteriores, y empiezan a ser mayores las diferencias por sexo $(38,39)$.

En nuestra población se observó que tanto el PT como el $A G B$ fue superior en las niñas, esto se debe fundamentalmente a que las niñas tienen mayor grasa corporal que los niños, debido a que las niñas experimentan cambios somáticos caracterizados por la aceleración en el crecimiento de la estatura, cambios en la composición corporal, sumado a reajuste del balance hormonal que da origen al establecimiento de la menstruación y la ovulación (40), mientras que en los niños es superior el valor del AMB (tabla 1), debido a un mayor incremento en sus tejidos magros en relación a las niñas. Estos resultados son similares a los del estudio realizado por Álvero-Cruz y col. (22) observando en una población de jóvenes entre 12 y 18 años, el porcentaje de masa grasa fue significativamente mayor en las niñas que en los niños, y además, la masa libre de grasa y el índice de masa libre de grasa (IMLG) fue mayor en los niños. Igualmente, Cuestas y col. (41) han observado que tanto el PT como la masa grasa fueron más elevados en las niñas.

Se analizó la presencia de obesidad central, para lo que se usaron la circunferencia de cintura y los índices IC/CA e ICA (42). La circunferencia de cintura y el IC/Ca son las medidas antropométricas utilizadas con frecuencia para la estimación de la grasa abdominal $(43,44)$ por correlacionarse positiva y significativamente con la cantidad de grasa intraabdominal y para identificar a las personas en riesgo cardiometabólico mejor que con el IMC únicamente (43). Se centró en el ICA debido a que ha adquirido relevancia estos últimos años por sus ventajas frente al perímetro abdominal (45), por ser el parámetro de adiposidad que mejor se asocia al síndrome metabólico (46), especialmente útil en poblaciones genéticamente predispuestas a la resistencia a la insulina (47), lo que refleja ser un predictor de factores de riesgo cardiovascular, así como diabetes tipo 2, hipertensión y dislipemia (48). En este estudio $24,1 \%$ de los escolares presentaron riesgo cardiovascular elevado por tener un ICA superior a 0,5. En el estudio realizado por Khoury y col. (49), en una población de entre 5 a 18 años, también lo observaron del 18\% al 72\%, dependiendo del factor de riesgo cardiometabólico el cual fue clasificado en 3 categorías $[<0,5,0,5 a<0,6 ; \geq 0,6]$, presentaron riesgo cardiovascular por tener un ICA superior a 0,6. Existen pocos estudios que hayan utilizado este índice, lo que pone de relieve la importancia de estudiarlo mejor (49).

Una de las limitaciones de nuestro estudio es que no se realizó la valoración del desarrollo puberal en adolescentes, considerando los índices de maduración sexual en cinco estadios (estadios de Tanner).

Un aspecto importante de este estudio es el gran tamaño de la muestra analizada.

\section{CONCLUSIÓN}

Nuestros resultados indican que a pesar de las campañas que se han realizado para disminuir el sobrepeso/obesidad, hay que seguir haciendo esfuerzos para disminuir estas cifras, pues siguen siendo elevadas. No se deberían descuidar los programas establecidos por el Ministerio de Sanidad y Consumo, que siguiendo las pautas de la OMS, están dirigidos a promover un estilo de vida saludable, además de procurar cambios en los hábitos de alimentación o actividad física, que también son términos que afectan al peso corporal y a la distribución de grasa corporal (50).

\section{RESUMEN}

Objetivo: Evaluar la prevalencia de sobrepeso y obesidad en una muestra de escolares españoles de acuerdo al sexo. Sujetos y métodos: Se estudiaron 505 escolares (259 varones y 246 mujeres), con edades entre 8 y 13 años $\left(4^{\circ}, 5^{\circ}\right.$ y $6^{\circ}$ de educación primaria), procedentes de diversas poblaciones españolas: A Coruña, Barcelona, Madrid, Sevilla y Valencia. Se recogieron los datos sanitarios, socioeconómicos, antropométricos (peso y talla) y de actividad física. Se calculó el Índice de Masa Corporal. Resultados: La prevalencia de sobrepeso de la población fue de $15,9 \%$, siendo mayor en las niñas $(21,9 \%)$ que en los niños $(10,1 \%)$, mientras que la de obesidad fue del $17,3 \%$, siendo mayor en los niños $(24,5 \%)$ que en las niñas $(9,7 \%)$. Un mayor porcentaje de grasa $(36,9 \%)$ medido por el área grasa del brazo (AGB\%) se observó en las niñas, y en cambio los niños presentaron mayor cantidad de grasa a nivel central medido por el índice cintura/cadera $(0,86 \pm 0,05)$. Conclusiones: Los escolares estudiados se encontraban en situación alarmante respecto al sobrepeso y obesidad. La situación ha empeorado con respecto a datos obtenidos en estudios previos, lo que pone de relieve la importancia de controlar éstos parámetros desde edades tempranas.

Palabras clave: Sobrepeso, obesidad, prevalencia, escolares, España.

Agradecimientos: Este artículo está basado en la tesis presentada por A. De Piero Belmonte en cumplimiento de los requisitos para el grado de Doctor por la Universidad Complutense de Madrid, España. La investigación fue financiada por un contrato Universidad-Empresa ( ${ }^{\circ}$ Expediente 210/2008) y una beca pre-doctoral de la Agencia Española de Cooperación Internacional para el Desarrollo (AECID).

\section{BIBLIOGRAFÍA}

1. Lama More, R. A., Alonso Franch, A., Gil-Campos, M., Leis Trabazo, R., Martínez Suárez, V., Moráis López, A., $y$ otros. Obesidad infantil. Recomendaciones del Comité de Nutrición de la Asociación Española de Pediatría Parte I. Prevención. Detección precoz. Papel del pediatra. An Pedriatr (Barc.) 2006;65(6):607-15.

2. Rodríguez-Rodríguez E., López-Plaza B., López-Sobaler A.,Ortega R. Prevalencia de sobrepeso y obesidad en adultos españoles. Nutr Hosp. 2011;26(2):355-63.

3. Fryar C.D., Carroll M., Ogden C. (2012). Prevalence of obesity among children and adolescents: United States, trends 1963-1965 through 2009-2010. Available from: http:// www.cdc.gov/nchs/data/hestat/obesity_child_09_10/ obesity_child_09_10.pdf

4. Kranz S, Mitchell D, Smiciklas-Wright H, Huang S, Kumanyika S, Stettler $N$ Consumption of recommended food groups among children from medically underserved communities. J Am Diet Assoc. 2009;109:702-7.

5. Ara I, Vicente-Rodríguez G, Moreno L, Gutin B, Casajus J. La obesidad infantil se puede reducir mejor mediante actividad física vigorosa que mediante restricción calórica. Apunts Med Esport. 2009;163:111-8.

6. AESAN (2011) Estudio de vigilancia del crecimiento "Aladino". Recuperado el 2/06/2012 de: http://www. naos.aesan.msssi.gob.es/naos/ficheros/investigacion/ ALADINO.pdf 
7. Müller-Riemenschneider F., Reinhold T., Berghöfer A., y cols. Health-economic burden of obesity in Europe. Eur J Epidemiol. (2008);23:499-509.

8. Oliva J., González L., Labeaga J.,y cols. Salud pública, economía y obesidad: el bueno, el feo y el malo. Gac Sanit. 2008;22:507-10.

9. Organización Mundial de la Salud (OMS). Comité de expertos de la OMS sobre el estado físico: uso e interpretación de la antropometría. Serie de informes técnicos, 854. Ginebra (Suiza) (1995).

10. Hernández M, Castellet J, Narvaíza JL, Rincón JM, Ruíz I, Sánchez E, y cols. Estudio longitudinal de crecimiento. Curvas de 0 a 8 años. Instituto de Investigaciones sobre crecimiento y desarrollo. Fundación F. Orbegozo. Madrid: Garsi. 1988.

11. Rubio MA, Salas-Salvadó J, Barbany M, Moreno B. Consenso SEEDO 2007 para la evaluación del sobrepeso y la obesidad y el establecimiento de criterios de intervención terapéutica. Med Clin (Barc). 2007;128:84-6.

12. Hernández M. Particularidades de la nutrición en la infancia: crecimiento y nutrición. En M Hernández Rodríguez, Alimentación infantil ( $3^{\circ}$ ed., págs. 3-12). Díaz de Santos 2001.

13. Freedman D. Relation of body fat patterning to lipid and lipoprotein concentrations in children and adolescents: The Bogalusa Heart Study. Am J Clin Nutr. 1989;50:930-9.

14. Ashwell M. Obesity risk: importance of the waist-to-height ratio. Nurs Stand J. 2009;23(41): 49-54.

15. Panjikkaran S, Kumari K. A. Augmenting BMI and waistheight ratio for establishing more efficient obesity. Percentiles among schoolgoing children. Indian J Community Med 2009;34(2):135-9.

16. Urbano JM. Tesis Doctoral. Validación de métodos antropométricos alternativos como marcadores de riesgo cardiovascular. Universidad de Extremadura. Facultad de Medicina. Departamento de Ciencias Biomédicas 2009.

17. Marrodán MD, Romero JF, Moreno S, Mesa MS, Cabañas MD, Pacheco JL, González-Montero M. Dinamometría en niños y jóvenes de entre 6 y 18 años: valores de referencia, asociación con tamaño y composición corporal. An Pediatr (Barc). 2009;70(4):340-8.

18. Ortega RM, Requejo AM, López-Sobaler AM. Modelos de cuestionarios para la realización de estudios dietéticos en la valoración del estado nutricional. En AM Requejo, RM Ortega, Nutriguía. Manual de nutrición clínica en atención primaria. Madrid: Complutense, 2006: p456-9.

19. Organización Mundial de la Salud (OMS). Requerimientos de energía y proteínas. Informe de un Comité de Expertos de la OMS. Serie de informes técnicos, 724. Ginebra (Suiza). 1985

20. IOM (2005b). Reference Intakes for energy, carbohydrate, fiber, fat, fatty acids, cholesterol, protein and aminoacids. Whasington, DC: The National Academies Press.

21. López de Lara D, Santiago P, Tapia M, Rodríguez M, Gracia $R$, Carrascosa A. Valoración del peso, talla e IMC en niños, adolescentes y adultos jóvenes de la Comunidad Autónoma de Madrid. An Pediatr (Barc). 2010;73(6):305-19.

22. Álvero-Cruz J, Álvarez E, Fernández J, Barrera J, Carrillo M, Sardhina L. Validez de los índices de masa corporal y de masa grasa como indicadores de sobrepeso en adolescentes españoles: estudio Escola. Med Clin (Barc). 2010;135(1):8-4

23. Velasco J, Mariscal-Arcas M, Rivas A, Caballero M, Hernández-Elizondo J, Olea-Serrano F. Valoración de la dieta de escolares granadinos e influencias de factores sociales. Nutr Hosp (2009);24(2):193-9.

24. MC Murray R, Harrell J, Deng S, Bradley C, Cox L, Bangdiwala $S$. The influence of physical activity, socioeconomic status, and ethnicity on the weight status of adolescents. Obes Res. (2000);8(2): 130-9.

25. Toledo E, Beunza J, Núñez J, Bes M, Basterra F, Martínez $M$. Metabolic risk factors in a cohort of young adults and their association with a body mass index between 22 and $25 \mathrm{~kg} / \mathrm{m} 2$. Med Clin (Barc). 2009;132:654-60.

26. Lara $M$, Bustos $P$, Amigo $H$, Silva $C$, Rona $R$. It waist circumference a better predictor of blood pressure, insulin resistance and blood lipids than body mass index in young children adults? BMC Public Health 2012;12:638.

27. Álvarez F, Díaz J, Riaño I, Pérez D, Venta $R$, Málaga S. Factores de riesgo cardiovascular clásico y emergentes en escolares asturianos. An Pediatr (Barc). (2011); 74 (en Prensa).

28. Durá T, Hualde J, Garralda I, Navarra GC. Exceso de peso corporal infantil en Navarra y su repercusión en la adolescencia. Med Clin (Barc). 2012;138(2):52-6.

29. Sánchez-Cruz J, Jiménez-Moleón J, Fernández-Quesada F, Sánchez M. Prevalence of child and youth obesity in Spain in 2012. Rev Esp Cardiol. 2013;66(5):371-6.

30. García García E, Vázquez López M, Galera Martínez R, Alias I, Martín González M, Bonillo Perales A, y cols. Prevalencia de sobrepeso y obesidad en niños y adolescentes de 2 a 16 años. Endocrinol Nutr. 2013;60(3):121-6.

31. Amigo I, Busto R, Pena E, Fernández C. Prevalencia de sobrepeso y obesidad en los niños de 9 a 10 años del Principado de Asturias: el sesgo de la valoración de los padres. An Pediatr (Barc.). (2013); 79 (en Prensa).

32. Rossen L. Neighbourhood economic deprivation explains racial/ethnic disparities in overweight and obesity among children and adolescents in the USA. J Epidemiol Community Health 2014; 68(2):123-9.

33. Heo M, Wylie-Rosett J, Pietrobelli A, Kabat G, Rohan T, Faith M. US pediatrics population-level associations of DXA-measured precentage of body fat with four BMI metrics with cutoffs. Internat J Obe. (2013); 38 (en Prensa).

34. Lozano Esteban M C. Tesis Doctoral. Condicionantes socioeconómicos de los hábotos alimentarios e ingesta de energía y nutrientes en escolares de la población española. Departamento de Nutrición y Bromatología I (Nutrición). Facultad de Farmacia. UCM. 2003.

35. Ortega P, Leal J, Amaya D, Chávez C. Evaluación nutricional, deficiencia de micronutrientes y anemia en adolescentes femeninas de una zona urbana y una rural del estado Zulia, Venezuela. Invest Clin. 2010;51(1):37-52.

36. Albañil Ballesteros M, Rogero Blanco M, Olivas Dominguez A, Sánchez Martín M, Rabanal Basalo A, Sanz Bayona M. Obesidad y factores de riesgo cardiovascular en adolescentes. Asociación con factores de riesgo cardiovascular en familiares de primer grado. Med Clin. 2012; 138(7): 283-8.

37. Alvarado J. Tesina Valoración antropométrica aplicada a la nutrición clínica. Programa de especialización tecnológica alimentaria. Carrera de Licenciatura en Nutrición. Ecuador. 2010.

38. Requejo A. Alimentación durante la fase de crecimiento estable: la etapa preescolar y escolar. En P Varela, Alimentación infantil. Aspectos de interés farmacéutico. ( $2^{\circ}$ ed.) Madrid: Novograf S.A. 1999; 109-16.

39. Muñoz M, Martí A. Dieta durante la infancia y la adoles- 
cencia. En J Salas Salvadó, A Bonada, R Trallero, M Engracia Saló, Nutrición y dietética clínica. Barcelona: Masson. 2000; 83-98.

40. Plaza, J. Puericultura de 0 a 20 años. Barcelona: Marbán Libros, S.L. 2010.

41. Cuestas E, Achával A, Garcés N, Larraya C. Circunferencia de cintura, dislipemia e hipertensión arterial en prepúberes de ambos sexos. An Pediatr (Barc). 2007;67(1):44-50.

42. Arnaiz $P$, Acevedo $M$, Díaz $C$, Bancalari $R$, Barja $S$, Aglony M, y cols. Razón cintura estatura como predictor de riesgo cardiometabólico en niños. Rev Chil Cardiol. 2010:29(3):281-8.

43. Fernández J, Redden D, Pietrobelli A, Allison D. Waist circumference percentiles in nationally representative samples of African-American, European-American, and Mexican-American children and adolescents. J Pediatr. 2004;145: 439-44.

44. Janssen I, Katzmarzyk P, Srinivasan S, Chen W, Malina $R$, Bouchard C, y cols. Utility of childhood BMI in the prediction of adulthood disease: Comparison of national and international references. Obes Res. 2005;13:1106-15.

45. Esmaillzadeh A, Mirmiran P, Azizi F. Comparative evaluation of anthropometric measures to predict cardiovascular risk factors in Tehranian adult women. Public Health Nutr. 2006;9:61-9.

46. Soto González A, Bellido D, Buño M, Pértega S, De Luis $D$, Martínez-Olmos M, y cols. Predictors of the metabolic syndrome and correlation with computed axial tomography. Nutrition 2007;23:36-45.

47. Misra A, Madhavan M, Vikram $N$, Pandey $R$, Dhingra $V$, Luthra K. Simple anthropometric measures identify fasting hyperinsulinemia and clustering of cardiovascular risk factors in Asian Indian adolescents. Metabolism 2006;55:1569-73.

48. Sung-Hee P, Soon-Ja C, Kwang-Soo L, Hyun-Young $P$. Waist circumference and waist-to-height ratio as predictors of cardiovascular disease risk in korean adults. Circ J. 2009;73:1643-50.
49. Khoury M, Manlhiot C, McCrindle B. Role of the waist/ heigh ratio in the cardiometabolic risk assessment of children classified by body mass index. J Am College Cardiol. 2013;62(8):742-51.

50. AESAN (2005). Estrategia NAOS: Estrategia para la Nutrición, actividad física y prevención de la obesidad. Ministerio de Sanidad y Consumo. Madrid. Recuperado el 2/06/2012 de:http://www.aesan.msc.es/AESAN/docs/ docs/publicaciones_estudios/nutricion/maqueta_NAOS1. pdf

51. Sánchez-Cruz J, Jiménez Moleón J, Fernández Quesada F, Sánchez M. Prevalence of child and youth obesity in Spain in 2012. Rev Esp Cardiol. 2013;66(5):371-76.

52. García García E, Vázquez López M, Galera Martínez R, Alias I, Martín González M, Bonillo Perales A, y cols. Prevalence of overweight and obesity in children and adolescents aged 2-16 years. Endocrinol Nutr. 2013:60(3):121-6.

53. Coronado Vázquez V, Odero Sobrado D, Canalejo González $D$, Cidoncha Pérez J. Prevalence of overweight and obesity in schoolchildren in rurañ areas. Gac Sanit. 2012;26(5): 460-2.

54. Ruíz Pérez L, Zapico Álvarez-Caseos M, Zubiaur Cantalapiedra A, Sánchez Paya J, Flores Serrano J. Increase in the prevalence of overweight and obesity in the pediatric population of the province of Alicante (Spain) in the last 10 years. Endocrinol Nutr 2008;55(9):389-95.

55. Ogden C, Carroll M, Curtin L, Mc Dowell M, Tabak C, Flegal $R$. Prevalence of overweight and obesity in the United State, 1999-2004. JAMA 2006:295(13):1549-55.

56. Albañil Ballesteros $M$, Sánchez Martín M, de la Torre Verdú M, Olivas Dominguez A, Sánchez Méndez M, Sanz Cuesta T. Prevalence of obesity in 14-years-olds in four primary care centers. Trends in weight changes since the age of two years old. An Pediatr (Barc.) 2005;63(1): 39-44.

57. Ene-Obong H, Ibeanu V, Onuoha N, Ejekwu A. Prevalence of overweight, obesity, and thinness among urban schoolage children and adolescents in southern Nigeria. Food Nutr Bull. 2012;33(4); 242-50. 